\title{
Pharmacokinetic Analysis of Montelukast in Healthy Korean Volunteers by High Performance Liquid Chromatography-Tandem Mass Spectrometry
}

\author{
Min-Ho Jo ${ }^{1}$, Mi-Sun Park ${ }^{1}$, Ji-Hyung Seo ${ }^{1,2}$, Wang-Seob Shim ${ }^{1}$, Sung-Vin Yim ${ }^{2}$ and Kyung-Tae Lee ${ }^{1,2 \nmid}$ \\ ${ }^{1}$ College of Pharmacy and \\ ${ }^{2}$ College of Medicine, Kyung Hee University, 1 Hoegi-Dong Dongdaemun-gu, Seoul 130-701, Republic of Korea \\ (Received July 27, 2011 · Revised August 1, $2011 \cdot$ Accepted August 8, 2011)
}

\begin{abstract}
A rapid and specific high performance liquid chromatography-tandem mass (LC/MS/MS) method for the analysis of montelukast in human plasma has been developed and validated. After cold acetonitrile-induced precipitation of the plasma samples, montelukast and glipizide (internal standard, IS) were eluted on a reverse-phase $\mathrm{C}_{18}$ column by isocratic mobile phase consisted of $10 \mathrm{mM}$ ammonium formate buffer (adjusted to $\mathrm{pH} 3.5$ with formic acid) and acetonitrile (3:97, $\mathrm{v} / \mathrm{v}$ ). Acquisition was performed with multiple reaction monitoring (MRM) mode by monitoring the transitions: $\mathrm{m} / \mathrm{z} 587.2 \rightarrow$ 423.2 for montelukast and $m / z \quad 446.0 \rightarrow 321.2$ for IS. Ranges of concentration for calibration curves $(10-1000 \mathrm{ng} / \mathrm{mL})$ showed correlation coefficients $\left(r^{2}\right)$ were better than 0.9948 . Precision of intra- and inter-day ranged from 3.70 to $11.68 \%$ and from 3.04 to $12.95 \%$, accuracy of intra-day and inter-day ranged from 93.34 to $102.75 \%$ and from 100.79 to $107.63 \%$, respectively. The described method provides a fast and sensitive analytical tool for determining montelukast levels in plasma, and was successfully applied to a pharmacokinetic study in 16 healthy human subjects after oral administration of $10 \mathrm{mg}$ tablet formulation of montelukast sodium under fasting conditions.
\end{abstract}

Key words - Montelukast, Pharmacokinetic study, Human plasma, LC/MS/MS, Korean

Montelukast, (S, E)-2-(1-((1-(3-(2-(7-chloroquinolin-2-yl) vinyl) phenyl)-3-(2-(2-hydroxy propan-2-yl) phenyl) propylthio) methyl) cyclopropyl) acetic acid, is antihistamic drug as a leukotrien receptor antagonist for using treatment of seasonal allergy and chronic asthma (Mastalerz et al., 2010; Castro-Rodriguez et al., 2010; Yasar et al., 2011; Schäper et al., 2011).

Several groups have reported methods for the determination of montelukast in biological matrices. These methods include chromatographic assay (Amin et al., 1995; Liu et al., 1997; AlRawithi 2001), usage of dual column (Smith et al., 2004), column switching (Ochiai et al., 1998), spectrofluorometry (Alsarra et al., 2005) or mass spectrometry (Challa et al., 2010; Sripalakit et al., 2008). However, most of these methods required laborious sample pretreatment or derivatization, and long chromatographic run times, and thus, are not convenient for analyzing the large numbers of samples generated during pharmacokinetic research. Thus, in the present study, our objective was to develop a rapid, sensitive, high throughput method for the routine determination of montelukast in human plasma.

Pharmacokinetic studies of montelukast were performed in various laboratories. In case of oral administration of $10 \mathrm{mg}$ film-coated tablet, it was reported the $\mathrm{T}_{\max }, \mathrm{AUC}_{0-\mathrm{t}}$ and $\mathrm{C}_{\max }$ was $3.7 \pm 0.8 \mathrm{hr}, 2441 \pm 441 \mathrm{ng} \cdot \mathrm{hr} / \mathrm{mL}$ and $385 \pm 85 \mathrm{ng} / \mathrm{mL}$, respectively (Cheng et al., 1996). Challa BR et al. reported pharmacokinetic research in 31 healthy Indians volunteers after oral administration of $5 \mathrm{mg}$ montelukast; $\mathrm{AUC}_{0-\mathrm{t}}, \mathrm{C}_{\max }$ and $\mathrm{T}_{\max }$ are decided to be $2417.26 \pm 63.58 \mathrm{ng} \cdot \mathrm{hr} / \mathrm{mL}, 369.29$ $\pm 137.35 \mathrm{ng} / \mathrm{mL}$ and $2.67 \mathrm{hr}$, respectively. Meanwhile other group (Sripalakit et al. 2008) investigated method for pharmacokinetic study of $10 \mathrm{mg}$ montelukast to 48 healthy Thai male volunteers. $\mathrm{AUC}_{0-t}, \mathrm{C}_{\max }$ and $\mathrm{T}_{\max }$ are presented each of $3712.41 \pm 1020.29 \mathrm{ng} \cdot \mathrm{hr} / \mathrm{mL}, 535.73 \pm 156.81 \mathrm{ng} / \mathrm{mL}$ and 3.61 $\pm 1.25 \mathrm{hr}$, respectively. Pharmacokinetic data for montelukast $10 \mathrm{mg}$ chewable tablet (Bharathi et al., 2009) were also found similar to those of tablet formation in human plasma; $\mathrm{C}_{\max }$ $(325 \pm 134.4 \mathrm{ng} / \mathrm{mL})$ was achieved at $3.50 \pm 0.00 \mathrm{hr}$ and the $\mathrm{AUC}_{0-\infty}$ was observed $3018.83 \pm 845.0 \mathrm{ng} \cdot \mathrm{hr} / \mathrm{mL}$.

Although pharmacokinetic studies of montelukast have been reported in various races, there has not been reported in the Korean populations. The purpose of the present study was designed to assess a pharmacokinetic study of montelukast in 16 healthy Korean volunteers who were administrated a $10 \mathrm{mg}$ tablet of montelukast sodium.

\footnotetext{
Corresponding Author:

Tel : +82-2-961-0860, E-mail : ktlee@khu.ac.kr

DOI : 10.4333/KPS.2011.41.5.289
} 


\section{Materials and Methods}

\section{Materials and reagents}

Montelukast sodium (99.8\% purity) was supplied from Daewoong Pharm. Co., Ltd. (Kyunggi-Do, Korea) and glipizide (internal standard, IS) was purchased Sigma-Aldrich (St.Louis, MO, USA), respectively. The structural formulas of montelukast (Figure 1A) and IS (Figure 1B) are shown. Ammonium formate and formic acid were also purchased from Sigma-Aldrich. Acetonitrile was obtained from J.T. Baker (Phillipsburg, USA). Water was obtained from aquaMAX ${ }^{\mathrm{TM}}$ ultra water purification system (YOUNGLIN, Kyunggi-Do, Korea). All other chemicals and reagents were of the highest analytical grade.
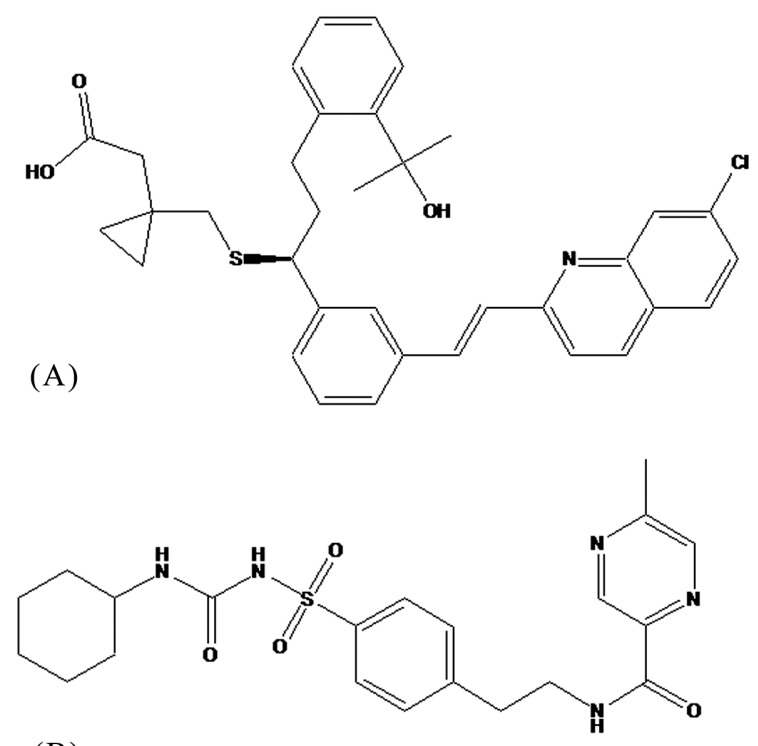

(B)

Figure 1. Chemical Structures of montelukast (A) and glipizide (B).

\section{LC/MS/MS condition for Montelukast in plasma samples} Chromatographic conditions

HPLC system was consisted of Agilent 1200 series model (Agilent Technologies, Waldbronn, Germany). LC separation was performed on Phenomenex luna $\mathrm{C}_{18}$ column $(50 \times 2.0 \mathrm{~mm}$, $3 \mu \mathrm{m})$ at $35^{\circ} \mathrm{C}$ with a mobile phase consisting of acetonitrile : ammonium formate buffer (adjusted to $\mathrm{pH} 3.5$ with formic acid $)=97: 3(\mathrm{v} / \mathrm{v})$, at a flow rate of $0.2 \mathrm{~mL} / \mathrm{min}$. The solution filtered using $0.22 \mu \mathrm{m}$ pore membrane filter and ultrasonically degassed prior to use.

\section{Mass spectrometric conditions}

Mass spectrometric detection was performed on API 4000 triple quadrupole mass spectrometer (Applied Biosystems MDS Sciex, Toronto, Canada) equipped in electro-spray ionization (ESI) positive ion mode. Injection for mass conditions was carried out using a solution of montelukast and IS, delivered at a constant flow-rate of $0.2 \mathrm{~mL} / \mathrm{min}$. The nebulizer and TurboIonSpray gases (nitrogen) were set at a value of 40 and $50 \mathrm{psi}$, respectively. The optimized TurboIonSpray voltage and temperature were set at $5500 \mathrm{~V}$ and $550^{\circ} \mathrm{C}$. Nitrogen was also used as curtain gas and collision gas, which were set at 14 and 6 psi. Quantification was performed using the multiple reaction monitoring (MRM) transition $\mathrm{m} / z$ 587.2 $\rightarrow \mathrm{m} / \mathrm{z} 423.2$ for montelukast, $m / z 446.0 \rightarrow m / z 321.2$ for IS, respectively (Figure 2). The optimized collision energy (CE) of $37 \mathrm{~V}$ was used for montelukast and $25 \mathrm{~V}$ for IS. The declustering potential (DP) was set at $57 \mathrm{~V}$ and $70 \mathrm{~V}$ for montelukast and IS, respectively. Analyst software (version 1.5) from Applied Biosystems MDS Sciex was used for data acquisition and handling.

Preparation of calibration standard and sample preparation The primary stock solution of montelukast $(1 \mathrm{mg} / \mathrm{mL}$ cal-

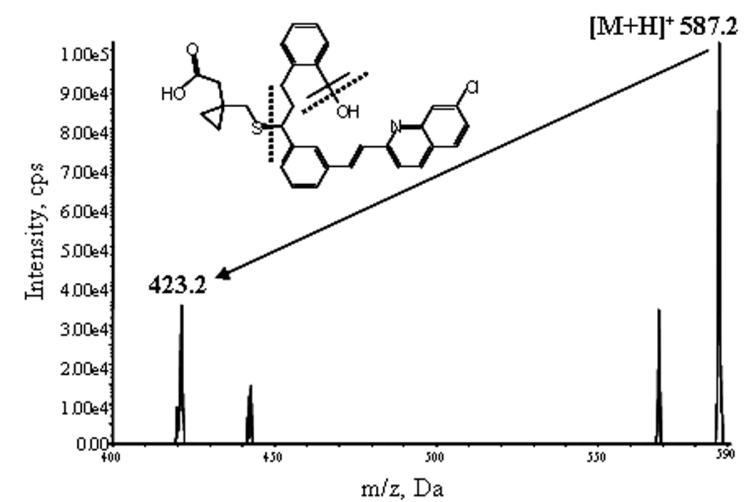

(A)

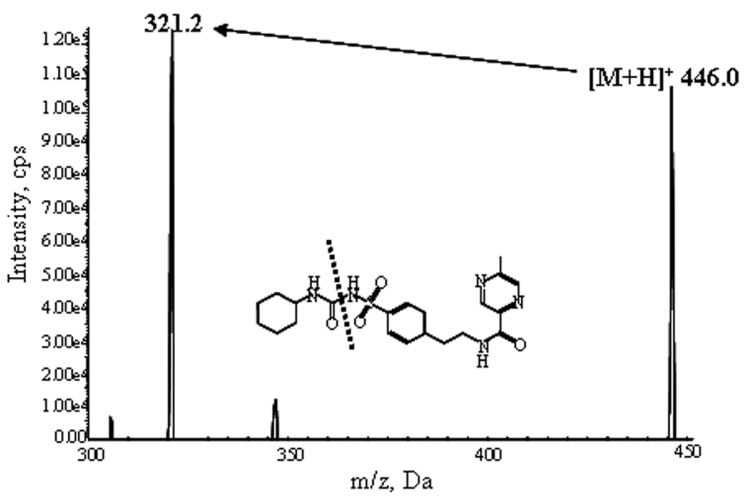

(B)

Figure 2. Product ion spectra of $[\mathrm{M}+\mathrm{H}]^{+}$ions of montelukast (A) and IS (B). 
culated as free base) was prepared in $100 \%$ acetonitrile. Standard stock solutions were diluted with acetonitrile to achieve concentration to $100,200,500,1000,5000$ and $10000 \mathrm{ng} / \mathrm{mL}$. IS primary stock solution was also prepared in dimethyl sulfoxide $(1 \mathrm{mg} / \mathrm{mL})$. IS stock solution was prepared to $3 \mathrm{ng} / \mathrm{mL}$ of solution diluted with acetonitrile. These were then stored at $-20^{\circ} \mathrm{C}$.

After thawing at room temperature and vortexing briefly, an aliquot of each sample $(100 \mu \mathrm{L})$ was added $35 \mu \mathrm{L}$ of IS $(3 \mu \mathrm{g} /$ $\mathrm{mL}$ ) and $600 \mu \mathrm{L}$ of cold acetonitlile. The mixture was vortexmixed for $15 \mathrm{~min}$ and then centrifuged at $14000 \mathrm{rpm}$ for 20 min at $4^{\circ} \mathrm{C}$. Each of sample supernatant $(150 \mu \mathrm{L})$ was filtered in syringe filter and then $5 \mu \mathrm{L}$ aliquot of the sample was injected onto the analytic column for LC-MS/MS analysis.

Montelukast is generally known as a light-sensitive compound and showed instability when exposed to light leading to the formation of its cis-isomer as the major photoproduct; ethylene moiety of montelukast readily changes from trans-form to cis-form (Al Omari et al., 2007). Therefore, we performed all procedures on sodium lamp based on the reports of advantage of sodium lamp.

\section{Method validation}

The method was validated for selectivity, linearity, sensitivity, and precision, accuracy according to the Korean Food and Drug Administration (KFDA) guidelines (KFDA, 2003) for validation of bioanalytical methods.

\section{Selectivity}

The selectivity of the method towards endogenous plasma matrix components was assessed in blank human plasma. Blank plasma samples from six different volunteers were tested for the presence of endogenous compounds, which might interfere with analytes, using the proposed extraction procedure and chromatographic/spectroscopic conditions. And then, it is compared with those obtained with a solution of the analyte at a concentration near the LLOQ.

\section{Calibration curve and linearity}

A calibration curve was constructed from six calibration samples covering the whole range $(10,20,50,100,500,1000$ $\mathrm{ng} / \mathrm{mL}$ ) by the peak area ratio of montelukast against IS. Concentration of montelukast was calculated from their area ratio with the calibration curve. The linearity of the calibration curve was also calculated. The calibration curve had to have a correlation coefficient $\left(r^{2}\right)$ of 0.99 or better. The limit of detection (LOD) was determined at a signal-to-noise $(\mathrm{S} / \mathrm{N})$ ratio of 3 , and the lowest standard on the calibration curve was to be accepted as the lower limit of quantitation (LLOQ), if the analyte response was at least 10 times more than that of drug free (blank) extracted plasma. In addition, the analyte peak of LLOQ sample should be identifiable, discrete, and reproducible with a precision $(\%$, C.V.) not greater than 20.0 and accuracy within $80.0-120.0 \%$.

\section{Precision and accuracy}

The intra- and inter-day assay precisions were determined as coefficient of variance $(\%$, C.V.), and intra- and inter-day assay accuracies were expressed as percentages of the theoretical concentration, as accuracy $(\%)=$ (found concentration/theoretical concentration $) \times 100$. Intra-day assay was performed with five replicates on the same day and inter-day assay was accomplished on four separate days. The acceptance criterion recommended by KFDA for each back-calculated standard concentration was $15 \%$ deviation from the normal value except LLOQ, which was set at $20 \%$.

\section{Pharmacokinetic study}

The proposed analytical method was applied to a pharmacokinetic study. A single oral dose of $10 \mathrm{mg}$ of montelukast was given to 12 healthy male volunteers. Blood samples were collected in tubes containing heparin before and after $0.5,1$, $1.5,2,2.5,3,3.5,4,5,6,8,12$ and $24 \mathrm{hr}$ of administration of drug. These were centrifuged at $3500 \mathrm{rpm}$ for $8 \mathrm{~min}$ to obtain plasma, and the supernatant plasma was put into micro-tube. These were then frozen at $-70^{\circ} \mathrm{C}$.

Pharmacokinetic parameters were calculated from plasma levels applying a non-compartmental statistic using BA calc 2002. Blood samples were drawn up to a period of three to five times the terminal elimination half-life $\left(t_{1 / 2}\right)$. The highest concentration $\left(\mathrm{C}_{\max }\right)$ and the time to reach the highest concentration $\left(\mathrm{T}_{\max }\right)$ values were determined by visual inspection of the plasma montelukast concentration-time curve $\left(\mathrm{AUC}_{0-\mathrm{t}}\right)$ obtained by the linear trapezoidal method in 0-24 hr.

\section{Results and Discussion}

\section{MS optimization and quantification}

MS/MS optimization was performed by directly infusing solutions of montelukast and glipizide (IS) into the electrospray injection (ESI) unit of a mass spectrometer at a constant flow-rate of $0.2 \mu \mathrm{L} / \mathrm{min}$. Quadrupole full scans (Q1 scans) were carried out in positive ion detection mode in order to optimize ESI conditions. The mass spectra of montelukast and glipizide revealed base peaks at $m / z 587.2$ and 446.0, respectively, which corresponded to the protonated molecular ions. 
The MS/MS condition parameters including collision gas, collision energy and collision cell entrance/exit potential were set to maximize the amounts of product ions produced. Product ion mass spectra and the fragmentation patterns of montelukast and glipizide are illustrated in Figure 2. The product ions of montelukast and glipizide were both at $\mathrm{m} / \mathrm{z} 423.2$ and $\mathrm{m} / \mathrm{z}$ 321.2. The instrumental parameters used are mentioned in the experimental section.

\section{Method validation}

\section{Selectivity}

No visible interferences were observed in the chromatograms of six blank plasma samples. Figure $3 \mathrm{~A}$ showed the chromatogram of a blank plasma sample with no endogenous peaks at the retention positions of montelukast or glipizide, which occurred at $c a .1 .40$ and $0.87 \mathrm{~min}$, respectively. The total run times for samples were ca.2.5 min (Figure 3).

\section{Calibration curve and linearity}

A six-point calibration curve exhibited good linearity for $\mathrm{LC} / \mathrm{MS} / \mathrm{MS}$ in the concentration range $10-1000 \mathrm{ng} / \mathrm{mL} \mathrm{ng} / \mathrm{mL}$. The linear regression equation of the analysis calibration curve was $y=0.004393( \pm 0.000065) x+0.005013( \pm 0.006571)\left(r^{2}\right.$ $=0.9964 \pm 0.00223)$. Where $y$ peak area is the area ratio of montelukast to IS, $x$ is the concentration ratio of montelukast to IS. The LLOQ for montelukast was proved to be $10 \mathrm{ng} / \mathrm{mL}$.

\section{Precision and accuracy}

The precision calculated as the C.V. and the accuracy was always below $15 \%$, other than near the LLOQ (below 20\%) in
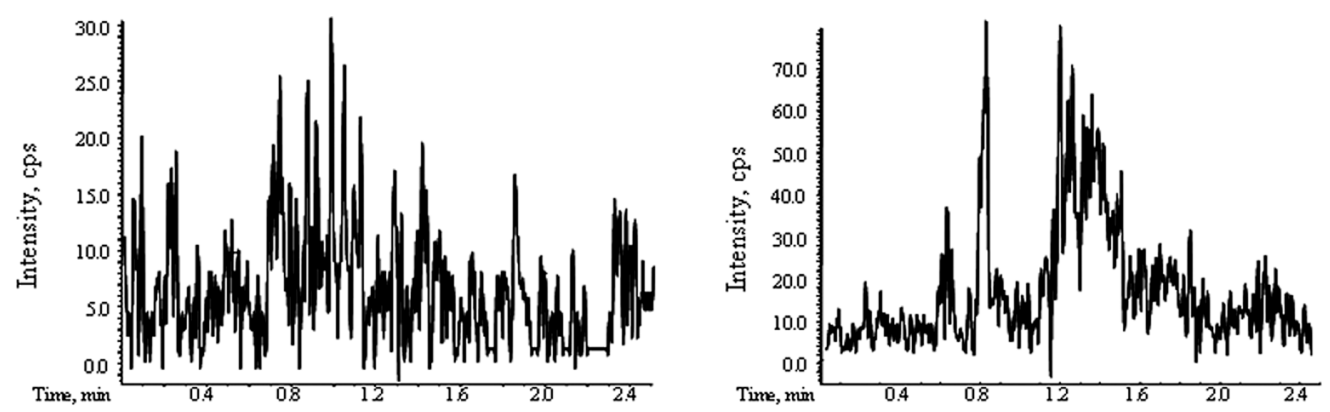

(A)
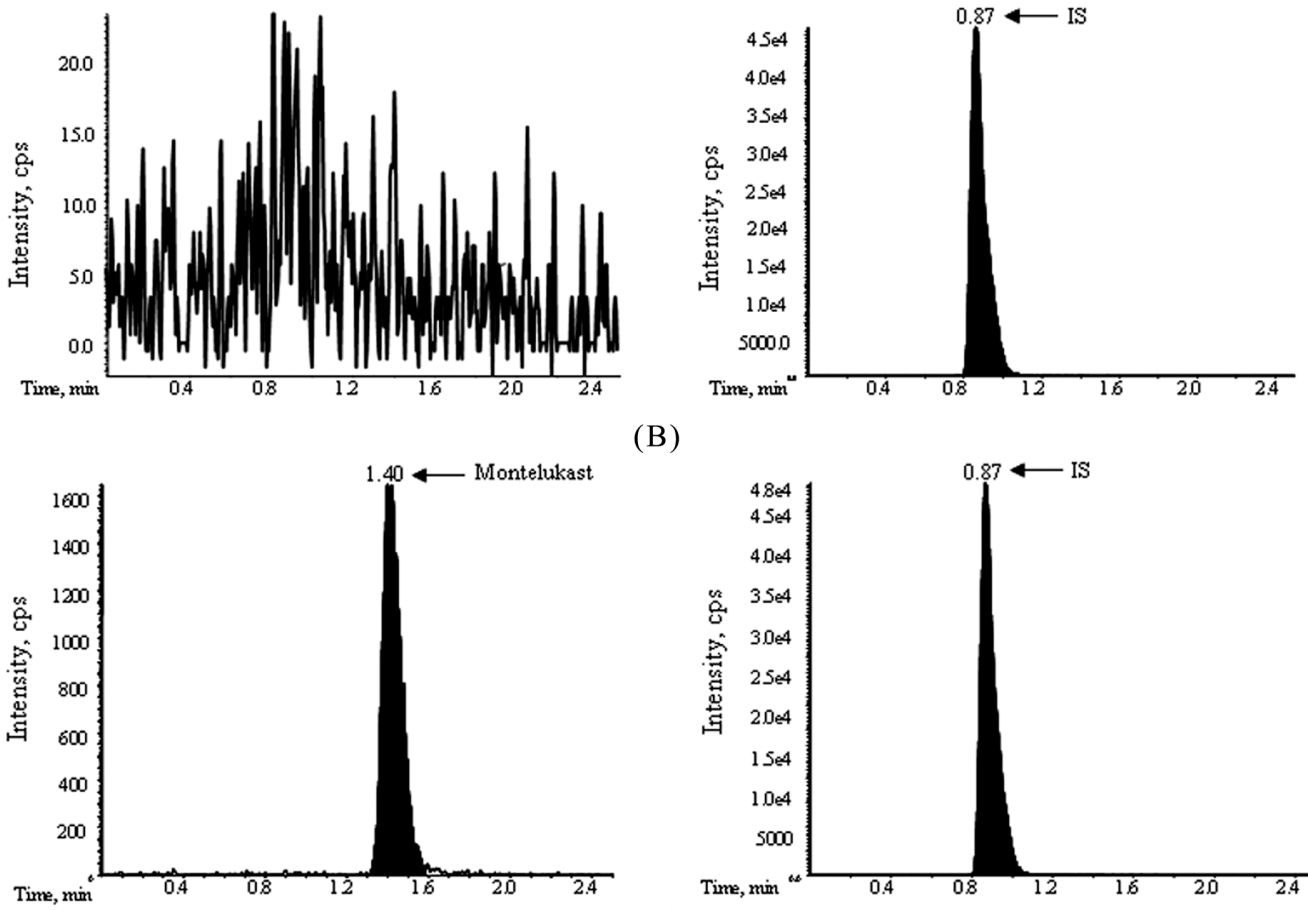

(B)

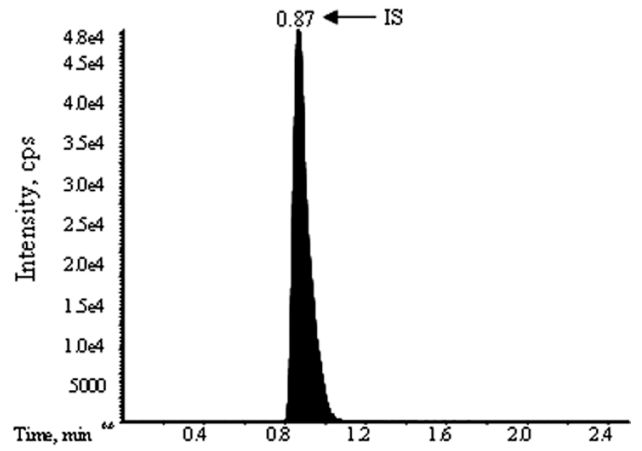

(C)

Figure 3. Chromatograms of the plasma blank (A), plasma blank spiked IS (B), plasma the lower limit of quantification (C). 


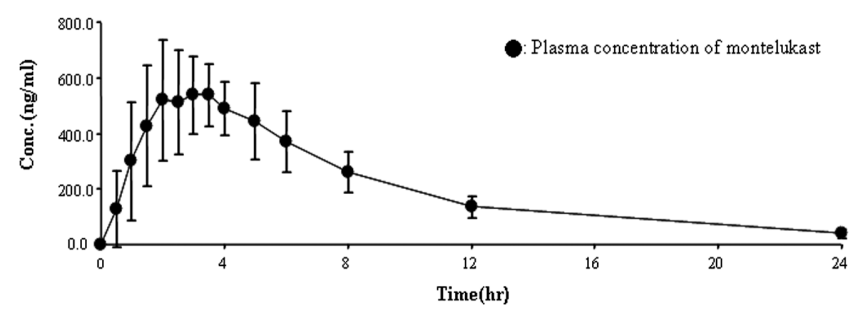

Figure 4. Mean $( \pm$ S.D, $n=16)$ plasma concentration-time curves of montelukast following oral administration of tablet (montelukast sodium $10 \mathrm{mg}$ ).

Table I. Precision and accuracy for the determination of montelukast in human plasma

\begin{tabular}{ccccc}
\hline \hline \multirow{2}{*}{$\begin{array}{c}\text { Concentration } \\
(\mathrm{ng} / \mathrm{mL})\end{array}$} & \multicolumn{2}{c}{ Precision (C.V. \%) } & \multicolumn{2}{c}{ Accuracy (\%) } \\
\cline { 2 - 5 } & Intra-day & Inter-day & Intra-day & Inter-day \\
\hline 10.00 & 12.95 & 3.70 & 100.79 & 96.68 \\
50.00 & 9.02 & 11.68 & 103.64 & 93.34 \\
500.00 & 3.04 & 9.04 & 107.63 & 102.75 \\
1000.00 & 7.38 & 6.52 & 104.69 & 98.26 \\
\hline
\end{tabular}

the concentration range from 10 to $1000 \mathrm{ng} / \mathrm{mL}$. Intra-day C.V.s of the method for montelukast ranged from 3.04\% to $12.95 \%$, while its accuracy ranged from $93.34 \%$ to $102.75 \%$. Inter-day C.V.s ranged from $3.70 \%$ to $11.68 \%$, and accuracies from $100.79 \%$ to $107.63 \%$ (Table I). These results satisfy KFDA criteria and confirm the reproducibility of this method. These results enable us to conclude that the devise method is well suited for routine high-throughput analyses and pharmacokinetic studies.

\section{Pharmacokinetic study}

The validated method was successfully applied for the determination of montelukast in 16 healthy male volunteers that orally administered $10 \mathrm{mg}$ of montelukast in tablet form. Fig. 4 shows a plasma concentration vs. time profile plot for montelukast in human subjects under fasting conditions. The method was sensitive enough to monitor montelukast concentrations for $24 \mathrm{hr}$. Approximately 350 samples including calibration, QC standards and volunteer samples were run and analyzed over 2 days, and the precisions and accuracies obtained for calibration and QC samples were well within acceptable limits. Percentage differences during sample reanalysis testing for assay reproducibility were less than $15 \%$. Mean area under the plasma-concentration versus time curve from 0 to $24 \mathrm{hr}\left(\mathrm{AUC}_{0-24}\right)$ after administration was $4910 \pm$ $916.391 \mathrm{ng} \cdot \mathrm{hr} / \mathrm{mL}$, and the mean area under the plasma-concentration curve from time 0 to infinity $\left(\mathrm{AUC}_{0 \text {-inf }}\right)$ was $5250.316 \pm 1012.806 \mathrm{ng} \cdot \mathrm{h} / \mathrm{mL}$. The observed mean maximum
Table II. Pharmacokinetic parameters obtained from the timeplasma concentration of montelukast after oral administration of $10 \mathrm{mg}$ montelukast sodium in 16 Korean male volunteers

\begin{tabular}{cccccc}
\hline \hline \multirow{2}{*}{ Subjuct } & \multicolumn{5}{c}{ Pharmacokinetics parameters } \\
\cline { 2 - 6 } $\mathrm{AUC}_{0-\mathrm{t}}$ & $\mathrm{AUC}_{0 \text {-inf }}$ & $\mathrm{C}_{\max }$ & $\mathrm{T}_{\max }$ & $\mathrm{T}_{1 / 2}$ \\
\hline 1 & 4637.895 & 5083.587 & 494.9 & 2 & 6.4 \\
2 & 5478.815 & 5652.399 & 980.2 & 1.5 & 4.8 \\
3 & 3658.415 & 3883.2 & 688.5 & 2 & 5.8 \\
4 & 6174.715 & 6832.171 & 612.9 & 4 & 6.3 \\
5 & 4522.650 & 4643.801 & 641.3 & 2.5 & 4.1 \\
6 & 5370.525 & 5895.324 & 557.0 & 1.5 & 6.8 \\
7 & 3688.260 & 4022.515 & 380.4 & 4 & 6.4 \\
8 & 3570.130 & 3714.309 & 496.7 & 2.5 & 4.6 \\
9 & 5780.995 & 6293.051 & 778.4 & 2.5 & 6.5 \\
10 & 5775.345 & 6181.728 & 841.8 & 2.5 & 6.2 \\
11 & 4383.190 & 4711.297 & 569.3 & 2 & 5.8 \\
12 & 4749.570 & 5050.893 & 697.8 & 2.5 & 5.9 \\
13 & 5737.960 & 6255.163 & 767.0 & 5 & 6.5 \\
14 & 5638.165 & 5769.146 & 702.4 & 3.5 & 4.1 \\
15 & 5766.150 & 6124.913 & 767.9 & 3.5 & 5.1 \\
16 & 3635.815 & 3891.557 & 748.9 & 1.5 & 3.0 \\
Mean & 4910.537 & 5250.316 & 670.3 & 2.7 & 5.5 \\
S.D & 916.391 & 1012.806 & 149.8 & 1.0 & 1.1 \\
\hline
\end{tabular}

plasma concentration $\left(\mathrm{C}_{\max }\right)$ was $670.3 \pm 149.8 \mathrm{ng} / \mathrm{mL}$, mean $\mathrm{T}_{\max }$ was $2.7 \pm 1.0 \mathrm{hr}$, and the half-life of montelukast during the terminal phase $\left(\mathrm{T}_{1 / 2}\right)$ was $5.5 \pm 1.1 \mathrm{hr}$ (Table II). Pharmacokinetic studies of montelukast have been reported in healthy human (Challa BR et al., 2010; Sripalakit P et al., 2008; Cheng H et al., 1996; Bharathi DV et al., 2009). Among the data, pharmacokinetic parameters are shown to similar property considering different administration dose. Although our study was determined somewhat higher AUC and $\mathrm{C}_{\max }$, a little difference in parameters between research groups may be made from the variation due to the method of determining drug concentrations, and individual variation; race, gastrointestinal motility or difference in metabolism. Therefore we could not find the significant difference in pharmacokinetic parameters of montelukast between races by the comparison of these parameters.

\section{Conclusions}

In this study, we have developed and validated more effective, rapid and simple method for determination montelukast in human plasma using LC-MS/MS. The devised method was 
also found to be satisfactory in terms of its selectivity, sensitivity, and reproducibility, and was successfully used in a pharmacokinetic study of montelukast administered orally as a single $10 \mathrm{mg}$ tablet in healthy Korean. This method may be useful for the pharmacokinetics and bioequivalence study of montelukast.

\section{References}

Challa, B.R., Awen B.Z., Chandu, B.R., Khagga, M., Kotthapalli, CB., 2010. Method development and validation of montelukast in human plasma by HPLC coupled with ESI-MS/ MS: Application to a bioequivalence study. Sci Pharm. 78(3), 411-422.

Sripalakit, P., Kongthong, B., Saraphanchotiwitthaya, A., 2008. A simple bioanalytical assay for determination of montelukast in human plasma: application to a pharmacokinetic study. J Chromatogr B Analyt Technol Biomed Life Sci. 869(1-2), 38-44.

Mastalerz, L., Kumik, J., 2010. Antileukotriene drugs in the treatment of asthma. Pol Arch Med Wewn. 120(3), 103-108.

Castro-Rodriguez, J.A., Rodrigo, G.J., 2010. The role of inhaled corticosteroids and montelukast in children with mild-moderate asthma: results of a systematic review with meta-analysis. Arch Dis Child. 95(5), 365-370.

Yasar, H., Kiran, B., Cagatay, T., Ozkul, H., Icten, S., 2011. The effect of montelukast sodium on serum arginase levels in patients with seasonal allergic rhinitis. Am J Rhinol Allergy.

Schäper, C., Noga, O., Koch, B., Ewert, R., Felix, S.B., Gläser S., Kunkel, G., Gustavus B., 2011. Anti-inflammatory properties of montelukast, a leukotriene receptor antagonist in patients with asthma and nasal polyposis. J Investig Allergol Clin Immunol. 21(1), 51-58.

Amin, R.D., Cheng, H., Rogers, J.D., 1995. Determination of MK0476 in human plasma by liquid chromatography. J Pharm Biomed Anal. 13(2), 155-158.

Liu, L., Cheng, H., Zhao, J.J., Rogers, J.D., 1997. Determination of montelukast (MK-0476) and its S-enantiomer in human plasma by stereoselective high-performance liquid chromatography with column-switching. J Pharm Biomed Anal. 15(5), 631-638.

Al-Rawithi, S., Al-Gazlan, S., Al-Ahmadi, W., Alshowaier, IA., Yusuf, A., Raines, D.A., 2011. Expedient liquid chromatographic method with fluorescence detection for montelukast sodium in micro-samples of plasma. J Chromatogr B Biomed Sci. 754(2), 527-531.

Smith, G.A., Rawls, C.M., Kunka, R.L., 2004. An automated method for the determination of montelukast in human plasma using dual-column HPLC analysis and peak height summation of the parent compound and its photodegradation product. Pharm Res. 21(9), 1539-1544.

Bharathi, D.V., Hotha, K.K., Jagadeesh, B., Mullangi, R., Naidu, A., 2009. Quantification of montelukast, a selective cysteinyl leukotriene receptor (CysLT1) antagonist in human plasma by liquid chromatography-mass spectrometry: validation and its application to a human pharmacokinetic study. Biomed Chromatogr. 23(8), 804-810.

Cheng, H., Leff, J.A., Amin, R., Gertz, B.J., De Smet, M., Noonan, N., Rogers, J.D., Malbecq, W., Meisner, D. Somers, G., 1996. Pharmacokinetics, bioavailability, and safety of montelukast sodium (MK-0476) in healthy males and females. Pharmaceutical Research. 13, 445-448.

Ochiai, H., Uchiyama, N., Takano, T., Hara, K., Kamei, T., 1998. Determination of montelukast sodium in human plasma by column-switching high-performance liquid chromatography with fluorescence detection. J Chromatogr B Biomed Sci Appl. 713(2), 409-414.

Alsarra, I., Khalil, NY., Sultan, M., Al-Ashban, R., Belal, F., 2005. Spectrofluorometric determination of montelukast in dosage forms and spiked human plasma. Pharmazie. 60(11), 823-826.

Al Omari, M.M., Zoubi, R.M., Hasan, E.I., Khader, T.Z., Badwan, A.A., 2007. Effect of light and heat on the stability of montelukast in solution and in its solid state. J Pharm Biomed Anal. 45(3), 465-471.

Korea FDA, 2003 Guideline for the Validation of Bioanalytical Method 2003.05. 